\title{
Thermally triggered on demand permeability of hybrid silica beds made of packed thermoresponsive organo-silica microparticles
}

\author{
S. A. Jadhav ${ }^{1,2^{*}}$, V. Spalletta ${ }^{1}$, D. Scalarone ${ }^{1}$ \\ ${ }^{1}$ Department of Chemistry and Nanostructured Interfaces and Surfaces (NIS) Research Centre, University of Torino, \\ Via P. Giuria 7, 10125 Torino, Italy \\ ${ }^{2}$ School of Nanoscience and Technology, Shivaji University Kolhapur, 416004 Kolhapur, Maharashtra, India
}

Received 3 June 2018; accepted in revised form 23 August 2018

\begin{abstract}
This work reports the preparation and testing of porous beds made from packed vinylated silica microparticles (SiMPs) grafted with thermoresponsive poly( $N$-isopropylacrylamide) (PNIPAM) and exhibiting on demand thermally triggered permeability. PNIPAM-grafted hybrid SiMPs with diameters of 1.40-1.55 $\mu \mathrm{m}$ were synthesized and characterized with Attenuated Total Reflection Fourier Transform Infrared Spetroscopy (ATR-FTIR), Thermogravimetric Analysis (TGA), Flow Particle Image Analysis (FPIA) and Scannning Electron Miscroscopy (SEM) to elucidate their composition and morphology. The PNIPAM-grafted SiMPs were used to prepare thermoresponsive beds of dimensions $1 \times 0.5 \times 0.5 \mathrm{~cm}$ inside filtration tubes. The thermo-regulated permeability of solutions of selected model compounds, namely caffeine, ketoprofen, orange II, bromocresol green and cresol red across the hybrid beds was tested by calculating the retention percentage of the model compounds at temperatures below and above the lower critical solution temperature (LCST) of PNIPAM. The results showed a clear control over the permeability of the packed hybrid beds mediated by external temperature changes. This control was achieved by virtue of the switchable nanovalves created by the thermoresponsive PNIPAM present in the inter-particle voids of the packed beds.
\end{abstract}

Keywords: smart polymer, silica microparticles, polymer grafting, hybrid thermoresponsive silica, thermo-regulated permeability

\section{Introduction}

Polymer-grafted solid or porous core particles are investigated with great interest for their use in adsorption [1], separation devices [2] and as components of hybrid membranes [3-5]. These hybrid particles can be packed into porous beds and they combine the intrinsic properties of the core particles and of the polymers grafted on them [6]. The particles used as a base for the preparation of porous beds provide mechanical stability while the polymers play an important role in modulating the surface properties of the beds. The efficiency of the porous beds based on polymer-grafted particles can be further enhanced by grafting stimuli responsive (smart) polymers that are able to change the surface properties in a reversible and controllable way. Till now such particles grafted with smart polymers have been investigated and tested with great interest for the development of drug delivery systems [7-9] but they can be of interest also for preparation of porous beds in environmental applications [10]. For instance, the grafting of thermoresponsive polymers such as poly $(N$-isopropylacrylamide) (PNIPAM) or its copolymers on the surface of stable materials results in hybrid materials with thermoresponsive properties which can be used to prepare smart porous beds for controlled transport 
of molecular species or selective absorption [11-15]. Upon heating above the lower critical solution temperature (LCST), the PNIPAM chains grafted on the particles undergo a typical reversible transition from water soluble hydrophilic coils to water insoluble hydrophobic globules [16-18]. This transition of the polymer transforms the silica surface into a surface with switchable properties, which is hydrophilic at normal (or room) temperatures and hydrophobic at elevated temperatures [19]. In addition, thermoresponsive polymers grafted on the surface of silica particles modify their packing behaviour and improve their stability [19].

In packed particle beds porosity is formed by the inter-particle voids. The presence of chemically grafted thermoresponsive polymers on the surface of the particles (i.e. on the pore walls) gives the possibility of having thermally triggered control over opening and closing of the voids by virtue of the typical thermo-reversible transition of the polymer. Such thermally controlled inter-particle voids are called as thermo-nanovalves [20]. Thus, filtration and separation systems based on thermoresponsive polymergrafted porous materials can help to create thermally controllable barriers at the solid-liquid or liquid-liquid interfaces. This control can be also obtained by complete blockage of the elution of solutions by closing the inter-particle nanovalves below the transition temperature of the polymer. These hybrid packed beds thus possess thermally triggered permeability with an open/close mechanism.

Thermoresponsive gating mechanisms applied to commercial porous membranes and porous materials with intrinsic porosity are studied with great interest [21]. This strategy is applied to control the permeability of solutions across the pores by using the open/ close switching function of the thermoresponsive polymers [22]. For this purpose, commercial porous glass or polymer membranes are used and a random grafting of themoresponsive polymers on the pores is carried out. Nevertheless this approach requires drastic treatments of the preformed or commercial porous membranes for the grafting of thermoresponsive polymers $[2,22,23]$. This may lead to the damage of the original porous structure or damage of the membrane itself. An alternative approach could be the preparation of packed particle beds as porous membranes made from the thermoresponsive polymer-grafted particles. As an important step towards this approach, in the present work, we report for the first time about preparation of a novel thermoresponsive hybrid silica bed cast inside filtration tubes whose permeability can be controlled by changing the temperature. First the synthesis of vinyl-functionalized silica microparticles (SiMPs) by modified Stober's process [24-29] was carried out, followed by the post-synthesis grafting of thermoresponsive PNIPAM by free radical polymerization technique. The packed particle beds were prepared inside filtration tubes by using the PNIAPM-grafted particles. Thermally trigged permeability of the solutions of selected model compounds across the beds was tested. An excellent control over the permeability was achieved making these newly synthesized hybrid beds an attractive system for various thermo-sensitive portable flow regulators, filtration and separation devices. A remarkable advantage of the approach is that the hybrid packed beds can be cast in any shape and inside any device with specific thickness.

\section{Experimental}

\subsection{Materials}

Vinyltrimethoxysilane (VTMS), $N$-isopropylacrylamide (NIPAM), potassium persulphate (KPS), $N, N$ methylenebisacrylamide (MBA), ethanol, 1,4-dioxane, ammonium hydroxide, caffeine (CF), ketoprofen (KP), orange II (OII), bromocresol green (BCG) and cresol red (CR), porous polyethylene frits and standard $3 \mathrm{ml}$ blank filtration tubes were purchased from Sigma-Aldrich, Italy. All the solvents used for the synthesis were of high purity and used as received.

\subsection{Instruments and methods}

Fourier-transform infrared (FTIR) spectra were recorded on a Perkin Elmer Spectrum 100 in the attenuated total reflectance (ATR) mode with a diamond crystal, using 32 scans per spectrum and a resolution of $4 \mathrm{~cm}^{-1}$ in the spectral range of 4000 $650 \mathrm{~cm}^{-1}$. DTGS was used as detector.

Thermogravimetric analyses (TGA) were carried out on a TA Q500 model from TA Instruments by heating samples in alumina pans at a rate of $10^{\circ} \mathrm{C} \cdot \mathrm{min}^{-1}$ from 25 to $600^{\circ} \mathrm{C}$ in a nitrogen flow and from 600 to $800^{\circ} \mathrm{C}$ in air. Change of the gas at $600^{\circ} \mathrm{C}$ was used to remove completely the carbonaceous residues from pyrolysis reactions and measure the exact amount of organic component.

A differential scanning calorimeter (DSC Q200, TA Inc.) was used to collect DSC thermograms of the samples and to determine the coil-to-globule transition 
temperature of PNIPAM. 5 mg of PNIPAM-grafted microparticles were dispersed in $5 \mathrm{ml}$ of water, a couple of drops were poured in an aluminium pan and analysed under nitrogen atmosphere by raising temperature from 20 to $60^{\circ} \mathrm{C}$ with a heating ramp of $10^{\circ} \mathrm{C} / \mathrm{min}$.

Flow particle image analysis (FPIA) was carried out using an automatic image analyser for the characterization of suspended particles in a range of 0.8 $80 \mu \mathrm{m}$ (Sysmex FPIA-3000, Malvern Instrument, Worcestershire, United Kingdom). The suspension is passed into a laminar flow through a measuring cell where, using a CCD camera and stroboscopic illumination, the instrument takes pictures of each particle. The analysis was carried out with the objective lens at $20 \times$ magnification in LPF (Low Power Field) mode to analyse particles of dimensions between 4 and $80 \mu \mathrm{m}$ or/and in HPF (High Power Field) mode for particles in the range from 0.8 to $20 \mu \mathrm{m}$. For each sample multiple analyses were performed on aliquots of $5 \mathrm{ml}$ of solution. Each aliquot was introduced into the instrument chamber, kept under stirring at a speed of $650 \mathrm{rpm}$ to ensure the maintenance of homogeneity and to avoid particle sedimentation. A volume of 5.5 microliters is automatically withdrawn from the suspension for analysis in LPF mode and $0.035 \mu \mathrm{l}$ for HPF. Each sampling was preceded by a sonication of 60 seconds to reduce aggregate formation and favour destruction of air bubbles.

Scanning electron microscope (SEM) images were obtained by a Zeiss EVO60 electronic microscope equipped with a Bruker Quantax200 XFlash ${ }^{\circledR 6} \mid 10$ SDD microprobe. Samples were dissolved in ethanol and poured onto an Al tape, and their analysis was performed in high vacuum at $15.00 \mathrm{kV}$. UV-Vis analyses were performed using a Lambda 25 spectrophotometer (Perkin Elmer). For quantification of the molecules used for testing of the temperature-regulated elution of model organic compounds, calibration curves were constructed by plotting absorbance vs. concentration between $10^{-5}$ and $10^{-8} \mathrm{M}(\varepsilon=$ $96043 \mathrm{M}^{-1}, R^{2}=0.997$ ) for caffeine, $10^{-1}$ to $10^{-6} \mathrm{M}$ ( $\left.\varepsilon=16311 \mathrm{M}^{-1}, R^{2}=0.999\right)$ for ketoprofen, $10^{-4}$ to $10^{-9} \mathrm{M}\left(\varepsilon=20196 \mathrm{M}^{-1}, R^{2}=0.999\right)$ for orange II, $10^{-2}$ to $10^{-6} \mathrm{M}\left(\varepsilon=30802 \mathrm{M}^{-1}, R^{2}=0.991\right)$ for bromocresol green and between $10^{-2}$ to $10^{-6} \mathrm{M}(\varepsilon=$ $15043 \mathrm{M}^{-1}, R^{2}=0.999$ ) for cresol red.

\subsection{Synthesis of SiMPs-PNIPAM}

\subsubsection{Step I: in situ synthesis of SiMPs-vinyl}

Vinyltrimethoxysilane (VTMS) $24 \mathrm{~g}$ was added drop by drop in $120 \mathrm{ml}$ deionized water in a single neck round bottom flask with vigorous stirring. The oil droplets of VTMS disappear completely by dissolution of the alkoxysilane and a transparent solution was obtained. Ammonium hydroxide $\left(\mathrm{NH}_{4} \mathrm{OH}, 30 \%\right.$, $2.5 \mathrm{ml}$ ) was added to the solution. After addition of the ammonia solution, the reaction mixture becomes turbid initially and immediately the formation of particles takes place. The reaction mixture was kept under magnetic stirring for $12 \mathrm{~h}$ at room temperature. After completion of the reaction, the vinyl-SiMPs were collected by centrifugation and washed twice with fresh D.I. water and acetone. The particles were then dried in oven at $40^{\circ} \mathrm{C}$ for several hours. The yield of particles was $12.01 \mathrm{~g}$.

\subsubsection{Step II: synthesis of PNIPAM-grafted SiMPs}

PNIPAM-grafted hybrid SiMPs were synthesized by radical copolymerization of vinyl-SiMPs with NIPAM monomer by using potassium peroxysulphate (KPS) as initiator. Solution of $0.4 \mathrm{~g}$ of KPS was prepared in $80 \mathrm{ml}$ of water. The suspension of vinyl-SiMPs $(4 \mathrm{~g})$ prepared in app. $15 \mathrm{ml}$ of ethanol was added to the solution of initiator. Next, the solution of NIPAM (4 g) and cross-linker $N, N$-methylenebisacrylamide (MBA, $0.4 \mathrm{~g}$ ) dissolved in $80 \mathrm{ml}$ water was added to the suspension of the particles containing the initiator. The reaction mixture was heated at $70^{\circ} \mathrm{C}$ for 16 hours with constant magnetic stirring. At the end of the reaction the polymer-grafted microparticles were recovered by centrifugation. The white residue of particles obtained was washed two times with water and acetone. Each time liquid phase was separated by centrifugation. The particles were then dried in oven at $40^{\circ} \mathrm{C}$ for several hours.

Two samples were prepared by changing the ratio (1:1 and 2:1) of monomer to vinyl functionalized silica particles. The polymer grafting obtained was 3.5 and $22 \%$ and the yields were 4.1 and $4.6 \mathrm{~g}$ respectively. (Refer to sample names as SiMPs-PNIPAM$3.5 \%$ and SiMPs-PNIPAM-22\% from here onwards). 


\subsection{Preparation and testing of particle packed beds}

The cartridges with PNIPAM-grafted microparticles beds embedded inside the small filtration columns were prepared by compressing the polymer-grafted particles between two commercially available porous polyethylene frits. Stable compact discs of dimensions $1 \times 0.5 \times 0.5 \mathrm{~cm}$ were formed by packing the particles together. The packed beds showed good mechanical stability. The solutions of specific concentration of model compounds, namely caffeine $\left(1.94 \cdot 10^{-3} \mathrm{~g} / \mathrm{l}\right)$, ketoprofen $\left(1.42 \cdot 10^{-2} \mathrm{~g} / \mathrm{L}\right)$ orange II $\left(3.5 \cdot 10^{-3} \mathrm{~g} / 1\right)$, bromocresol green $\left(6.98 \cdot 10^{-4}\right)$ and cresol red $\left(9.6 \cdot 10^{-3}\right)$ were allowed to pass across the beds at 20 and $40^{\circ} \mathrm{C}$. These two temperatures are below and above the LCST of the tethered PNIPAM $\left(33.53^{\circ} \mathrm{C}\right)$ on the particles as measured by DSC analysis. The permeation was accelerated by connecting the other end of the filtration tube to a peristaltic pump. The flow rate was adjusted to $3 \mathrm{ml}$ per minute. The eluents $(3 \mathrm{ml})$ were collected and filtered before analysis. The concentrations of the molecules were monitored by UV-visible spectroscopy.

\section{Results and discussion}

\subsection{Synthesis of hybrid PNIPAM-grafted SiMPs}

The hybrid PNIPAM-grafted microparticles used in this study were synthesized by following the two step synthetic protocol shown in the Figure 1. The first step is the synthesis of vinyl functionalized silica microparticles which was carried out by modified Stober's process by the hydrolysis of VTMS in basic medium [30]. In the second step the growth of PNIPAM on vinyl functionalized particles was carried out by radical polymerization of NIPAM monomer by using potassium persulphate (KPS) as initiator. KPS is a versatile radical initiator which works well in various conditions to synthesize linear and cross-linked PNIPAMs [31-34]. Here MBA was used as the cross-linking agent [35]. The process resulted in PNIPAM-grafted silica microparticles. These hybrid PNIPAM-grafted particles were fully characterized by ATR-FTIR, TGA and, FPIA and SEM to confirm the effective grafting of the PNIPAM and to check the quantitative grafting of the polymer. The vinyl-functionalized silica particles to monomer (NIPAM) ratio was changed from 1:1 to $1: 2$ to prepare two samples with low (3.5\%) and intermediate (22\%) PNIPAM grafting respectively.

\subsection{Characterization of hybrid particles \\ 3.2.1. ATR-FTIR}

The ATR-FTIR spectra of vinyl functionalized SiMPs, and SiMPs-PNIPAM with 3.5 and $22 \%$ polymer grafting are shown in Figure 2. The spectrum of vinyl functionalized SiMPs showed the characteristic absorption peak of $\mathrm{C}=\mathrm{C}$ group at $1607 \mathrm{~cm}^{-1}$, the peak at $1413 \mathrm{~cm}^{-1}$ due to $\mathrm{C}-\mathrm{H}$ bending and the typical very broad peak due to the $\mathrm{Si}-\mathrm{O}-\mathrm{Si}$ stretching vibrations between 990 to $1072 \mathrm{~cm}^{-1}[36,37]$. The $\mathrm{Si}-\mathrm{O}-\mathrm{Si}$ peak confirmed the siloxane network (particle) formation while the vinyl group signals confirmed their functionality. Instead, both samples of PNIPAM-grafted SiMPs showed typical absorption peaks for the polymer (PNIPAM) at 1385, 1479 and at 1566, $1667 \mathrm{~cm}^{-1}$ which are attributed to $\mathrm{C}-\mathrm{H}$ bending typical of the isopropyl group, $-\mathrm{CH}_{3}$ bending, $\mathrm{C}-\mathrm{N}$ (amide) stretching and $\mathrm{C}=\mathrm{O}$ (amide) stretching, respectively [3841]. It is worth pointing out that the intensities of the peaks due to the presence of the polymer were considerably increased with increased grafting from 3.5 to $22 \%$. These results indicating increased PNIPAMgrafting were further confirmed by TGA analysis.

\subsubsection{TGA and DSC}

TGA analyses of the vinyl functionalized and PNIPAM-grafted SiMPs were carried out to confirm the presence of the polymer and to determine the quantitative grafting. The TGA curves of all samples

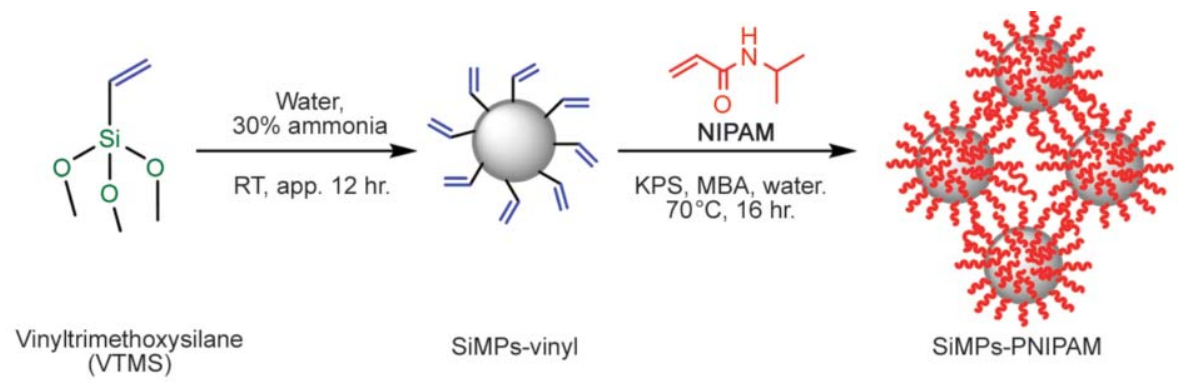

Figure 1. Synthesis protocol for in situ vinyl-functionalized SiMPs from VTMS and PNIPAM-grafted SiMPs. 

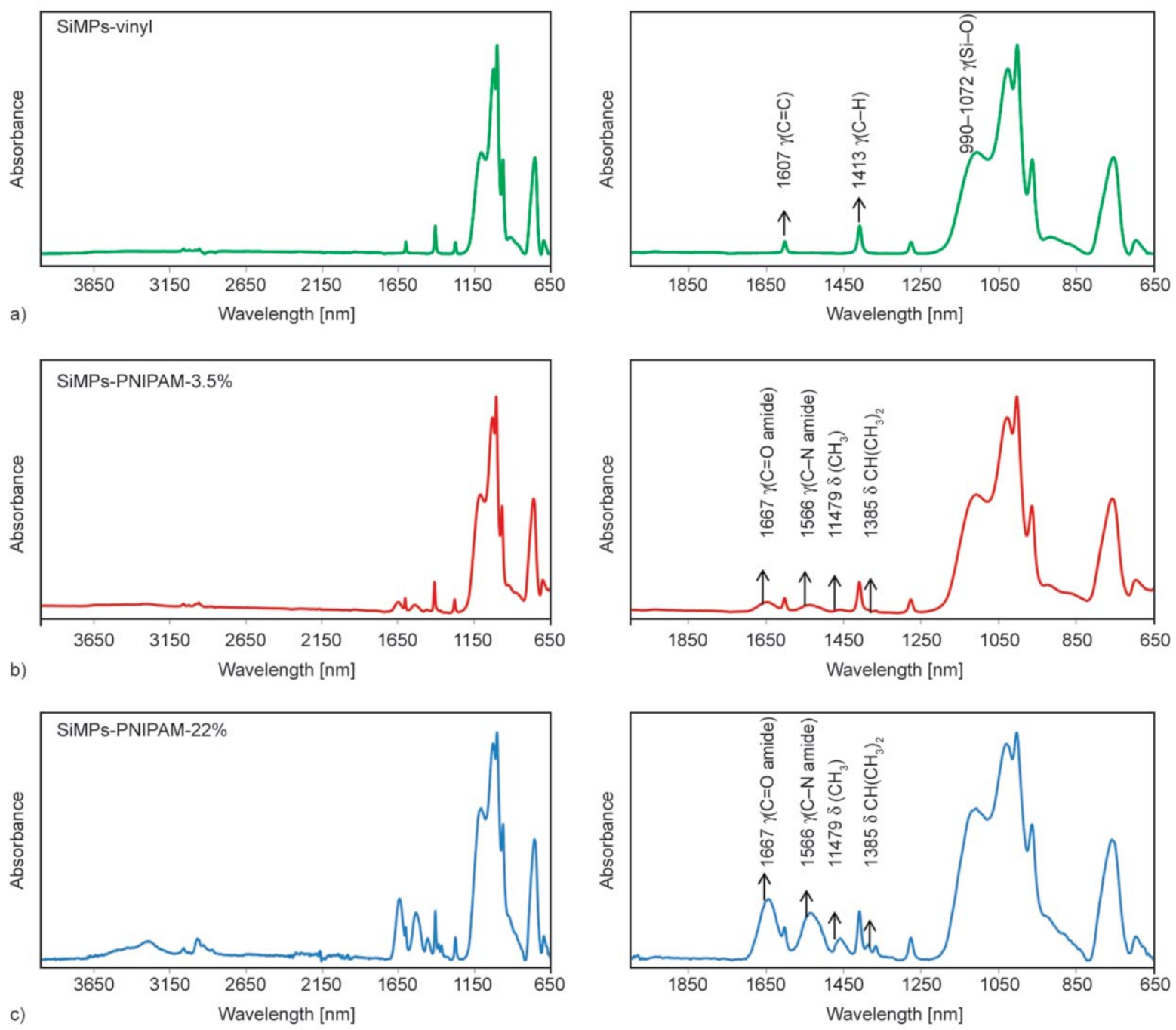

Figure 2. ATR-FTIR spectra of a) SiMPs-vinyl, b) SiMPs-PNIPAM-3.5\% and c) SiMPs-PNIPAM-22\%. Zoomed areas of each spectra are shown on the right hand side.

are shown in Figure 3. The samples SiMPs-vinyl, SiMPS-PNIPAM-3.5\% and SiMPs-PNIPAM-22\% showed the total weight loss of $24.5,28$ and $46.5 \%$ respectively. The initial heating of the samples $(25$

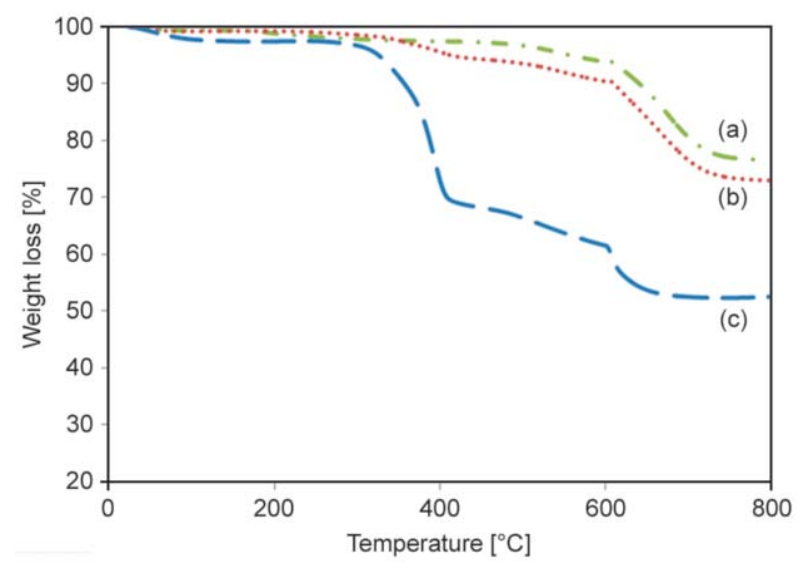

Figure 3. TGA curves of (a) SiMPs-vinyl (green dash dot line), (b) SiMPs-PNIPAM-3.5\% (red dotted line) and (c) SiMPs-PNIPAM-22\% (blue long dash line). to $\left.600^{\circ} \mathrm{C}\right)$ was carried out in nitrogen and later $(600$ to $800^{\circ} \mathrm{C}$ ) in air to fully degrade the organic component. No complete degradations of samples were observed up to $800^{\circ} \mathrm{C}$, which indicates the good stability of the core of the particles prepared by the precipitation route. The weight percentage of grafting of PNIPAM on the vinyl-functionalized particles was calculated by subtracting the weight loss due to the vinyl-functionalized particles to the weight loss of the the SiMPs-PNIPAM samples. The percentage grafting of PNIPAM on the two SiMPs samples was calculated and it was to 3.5 and $22 \%$ respectively. Thus, the two samples have a considerably different polymer-grafting, and the characterization and testing of their thermo-regulated permeability allows understanding the effect of the amount of PNIPAM on the capability to control permeability.

The transition temperature of the PNIPAM-grafted sample, which was found to be around $33.53^{\circ} \mathrm{C}$, was 


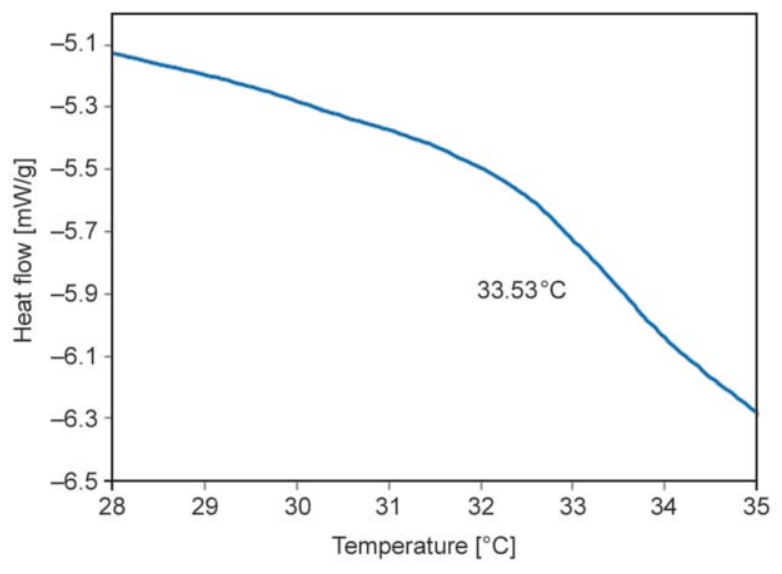

Figure 4. DSC curve of SiMPs-PNIPAM showing transition temperature.

determined by DSC analysis (Figure 4). Based on this observation, the testing of permeability of the porous beds prepared from these particles was carried out at 20 and $40{ }^{\circ} \mathrm{C}$ as these temperatures are well below and above the LCST of the polymer.

\subsubsection{FPIA and SEM}

The FPIA of vinyl functionalized and PNIPAMgrafted particles were carried out on stable suspensions prepared in deionized water and the results are shown in Figure 5. It is important to measure the hydrodynamic diameter (HD) of the particles before their use in real applications. The HD in particular gives an idea of exact effective diameter of the particles when they are suspended in water. The results showed the formation of stable vinyl functionalized silica microparticles with narrow particle size distribution (PSD) (Figure 5a) and with average diameter of $1.38 \mu \mathrm{m}$. Although, the precipitation route synthesis is a rather easy method of synthesis of silica particles, a strict control over the reaction parameters
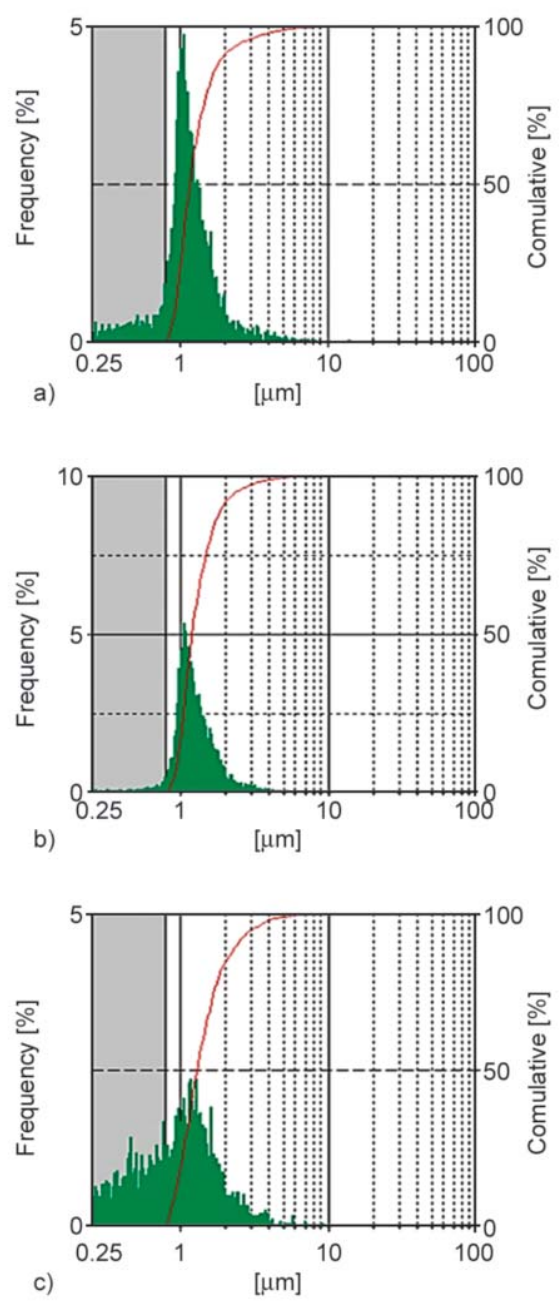

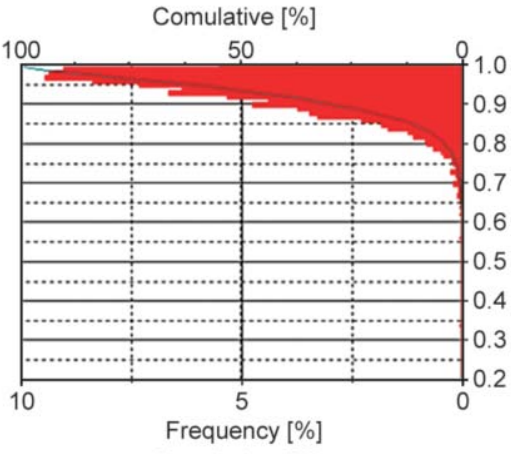

Comulative [\%]
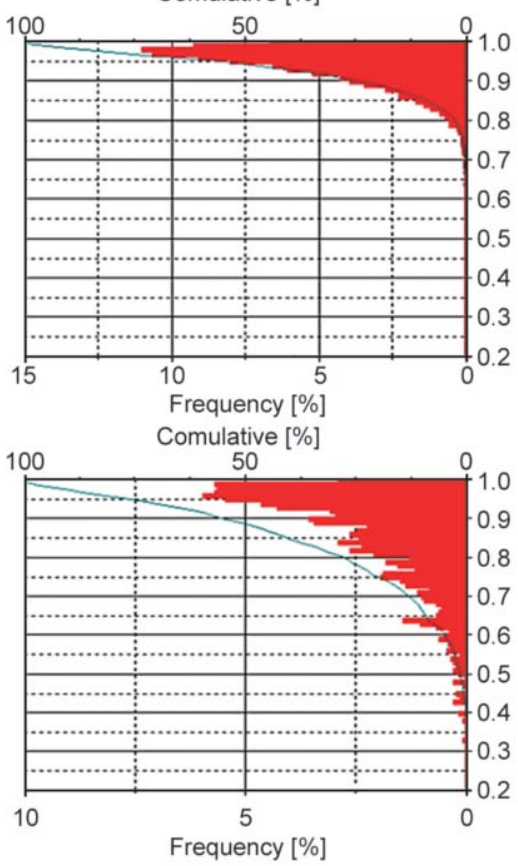
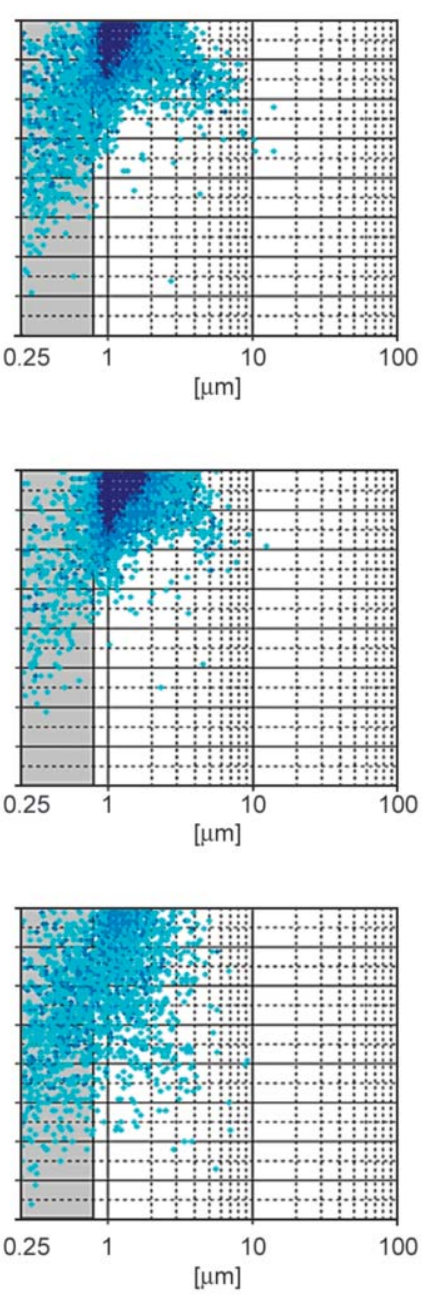

Figure 5. FPIA results of a) SiMPs-vinyl, b) SiMPs-PNIPAM-3.5\%, c) SiMPs-PNIPAM-22\%. In the order from right to left: Particle size distribution, circularity and scatterogram of size and circularity. 
is necessary to obtain particles with narrow PSD. This was achieved by suitable modifications of the previously reported methods [35]. The average size of the particles with 3.5 and 22\% PNIPAM grafting was increased to 1.40 to $1.55 \mu \mathrm{m}$ respectively. This was due to the increased amount of polymer coating on the microparticles. These results supported the observations made by IR and TGA analysis about qualitative and quantitative PNIPAM-grafting on the particles. The PSD of particles was found to become broader upon increased polymer grafting $(22 \%)$; this may be due the increased inter-particle crosslinking due to the high amount of polymer. Furthermore, the HD values determined by FPIA provide an indirect measure of the variation of the particles size associated with the coil-to-globule transition of PNIPAM chains. Assuming that PNIPAM chains in globular conformation, i.e. collapsed on the surface of the microparticles, do not contribute significantly to the diameter of the particles, it can be estimated that the variation of HD associated to the coil-to-globule transition is $20 \mathrm{~nm}$ for the microparticles with 3.5\% PNIPAM grafting and $170 \mathrm{~nm}$ for the microparticles with $22 \%$ PNIPAM grafting. It is interesting to note that the first of the two data is comparable with the reported data [8]. Another important feature of the particles synthesized was the higher circularity index. The cumulative percentage of the perfectly circular particles was almost $100 \%$ for all the samples. It showed the formation of good stable spherical SiMPs under the reaction condition chosen. More importantly the solid silica core remained stable in the polymerization conditions. Also the PNIPAM-grafting showed no effect on the circularity of the particles. The formation of stable perfectly spherical particles was further confirmed by recording the SEM images of vinyl-functionalized and PNIPAM-grafted particles. The SEM (Figure 6) images of the SiMPs-vinyl, SiMPs-PNIPAM 3.5 and $22 \%$ grafted-particles are shown in Figures $6 a, 6 b$ and $6 c$ respectively. The presence of discrete spherical particles and slight changes in their appearance upon PNIPAM-grafting was observed.

\subsection{Preparation of beds inside filtration tubes and testing}

Porous membranes made from the thermoresponsive hybrid particles were prepared by packing the PNIPAM-grafted silica microparticles inside small filtration tubes. The imaginary possible packing of

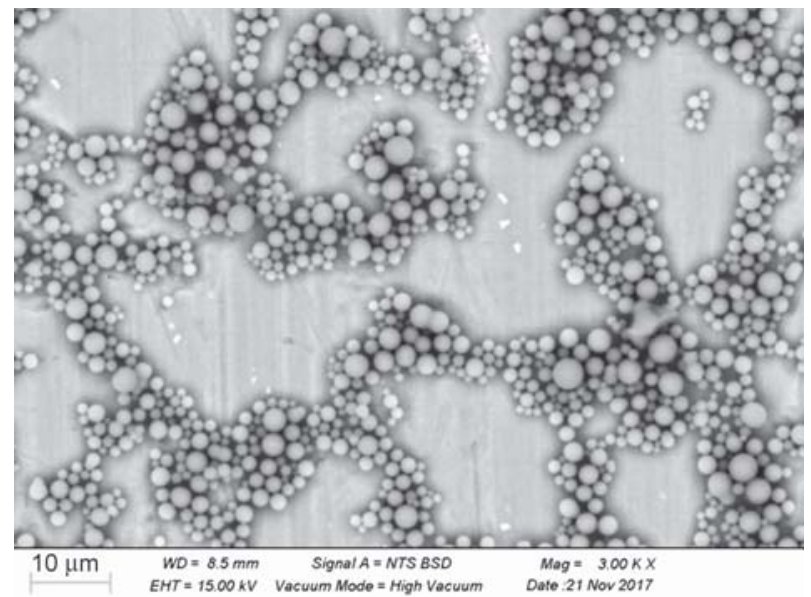
a)

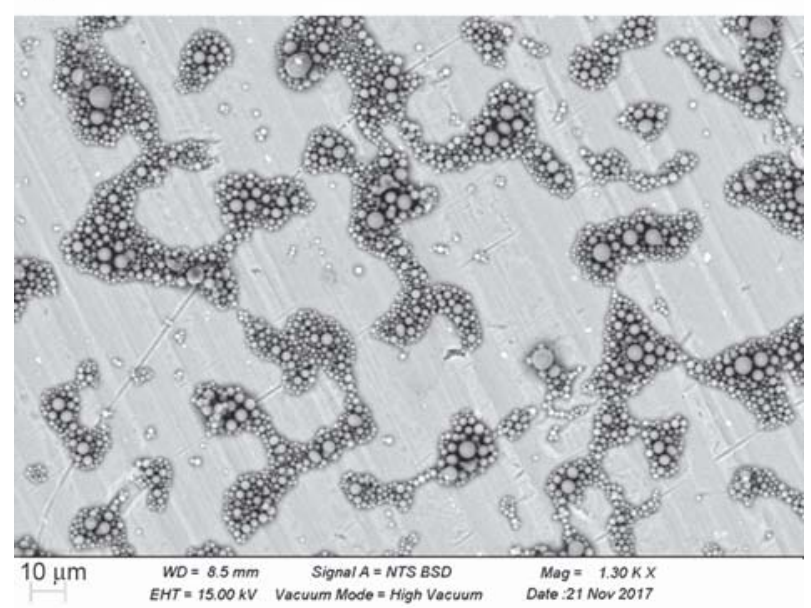

b)

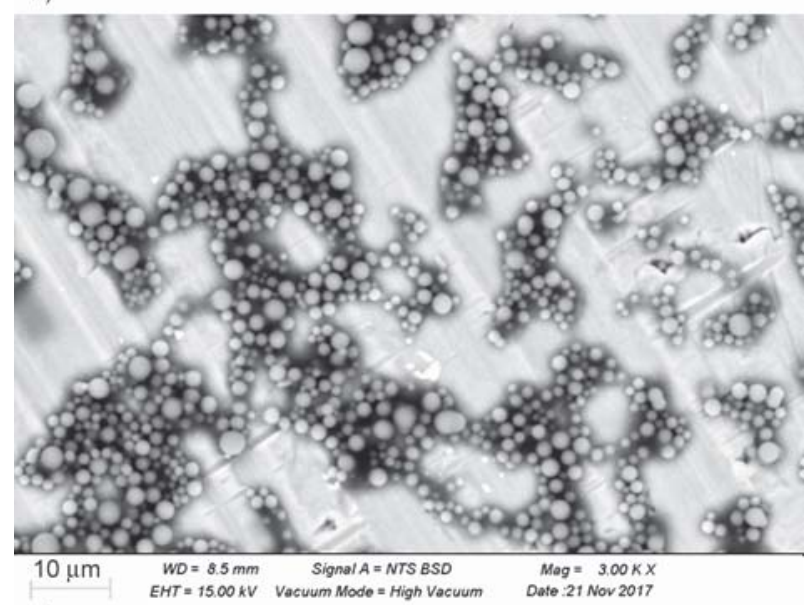

c)

Figure 6. SEM images of a) SiMPs-vinyl, b) SiMPs-PNIPAM-3.5\%, c) SiMPs-PNIPAM-22\%.

PNIPAM-grafted silica microparticles inside the bed is illustrated in Figure 7. The inter-particle voids are occupied by the PNIPAM chains. When the hybrid silica bed is wet with water solutions at room temperature (or below the LCST of the polymer) PNIPAM chains, which are in the extended and hydrated form, block the permeability of the solution of molecules 


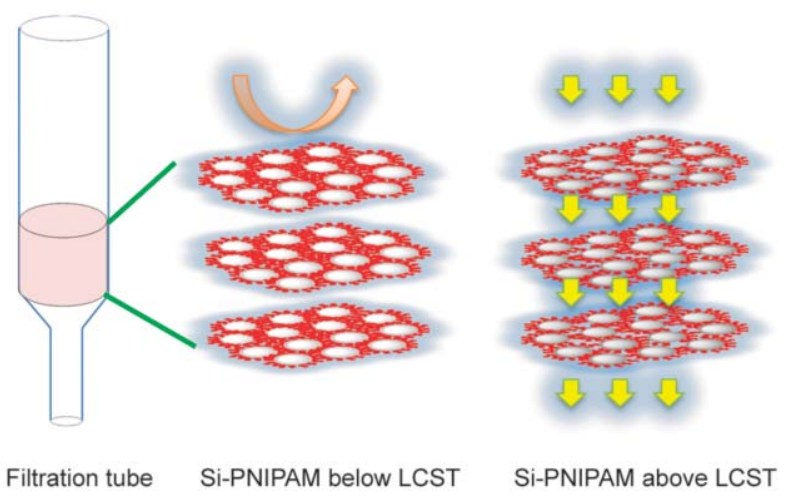

Figure 7. Illustration of imaginary packing of PNIPAMgrafted silica microparticles inside porous beds and thermoresponsive transition of the polymer in the inter-particle voids at below and above the LCST of the polymer.

across them. In addition, hydrophobic vinyl groups are exposed to the water solutions and they help to prevent the permeability. As the temperature is raised above the LCST of PNIPAM the polymer chains shrink to form compact globules on the particles surface, thus shielding vinyl groups and opening the nanovalves which trigger the permeability of the solutions across the beds. Thus the responsive polymer coating on the particles also protects the particles by preventing the contact between water solutions of various compounds and the particle surface at elevated temperatures. The same experiments carried out with the packed beds prepared from bare silica microparticles (not PNIPAM-grafted) resulted in complete blockage of solutions both at room temperature and at $40^{\circ} \mathrm{C}$. It is also worth pointing out that the particle-packed column system prepared, thus eventually became a particle-packed composite membrane system with thermoresponsive gating mechanism.

The results of the permeability tests and concentrations of the eluents in the form of percentage retention of the five model compounds across the porous beds prepared from SIMPs samples with 3.5 and $22 \%$ PNIPAM grafting are reported in Figure 8 . The details of the concentrations of the starting solutions and the concentrations of the solutions eluted across the beds are presented in Table 1. The choice of the model molecules to test the permeability was based upon their UV-visible activity and coverage of the molecular weight range from app. 200 to $800 \mathrm{~g} / \mathrm{mol}$. The retention of solutions of all molecules by the porous beds below the LCST of PNIPAM was $100 \%$ that is the beds showed complete blocking of the pores and zero permeability. As already explained this was due to the presence of polymer chains in their fully extended and hydrated conformation, which partially obstruct the pores, and to the intrinsic hydrophobicity of vinyl groups. As the temperature is raised above the LCST of the polymer $\left(33.53^{\circ} \mathrm{C}\right)$ the permeability of the bed is increased and the elution of the solutions across the beds is possible due to the opening of the valves. Different percentages of release were obtained depending on the secondary interactions of the various functional groups present on the model compounds with the polymer. In general, it was noticed that the SiMPs-PNIPAM sample with $3.5 \%$ polymer grafting showed remarkable control over the permeability and gating mechanism with respect to the sample with higher $(22 \%)$ polymer grafting. This proved that the higher amount of polymer grafting was ineffective in governing the gating mechanism possibly due to the formation of uneven porous voids and inter-linking of the particles. The percentage of retention by porous beds prepared from SiMPs-PNIPAM-3.5\% sample for CF, KP, O-II, BCG, and CR was $63,42,34,4$ and $0 \%$ respectively. While the beds prepared from SiMPsPNIPAM-22\% sample showed uneven retention and also very high values of retention percentage (39$100 \%$ ) indicating the altered particle packing behavior upon high polymer grafting and possibly also the formation of considerably big size collapsed (dehydrated) structures of the polymer blocking the interparticle voids. Both these points collectively resulted in higher retention of model compounds even at elevated temperature on the porous beds prepared from the SiMPs-PNIPAM-22\% sample. It is important to point out here that at elevated temperatures the beds showed improved or complete permeability for the solutions used. Instead, the change in concentrations of the starting solutions is due to the adsorption of certain amount of the compounds on the beds (silica surface). Hence the decrease in concentrations is not related to the permeability directly.

\section{Conclusions}

In this work, PNIPAM-grafted silica microparticles were synthesized and characterized. Porous packed particle beds were prepared from the polymer-grafted particles and tested for their thermosensitive permeability. The thermo-regulated permeation of the solutions of model compounds across the beds was studied and the results proved that the PNIPAM 

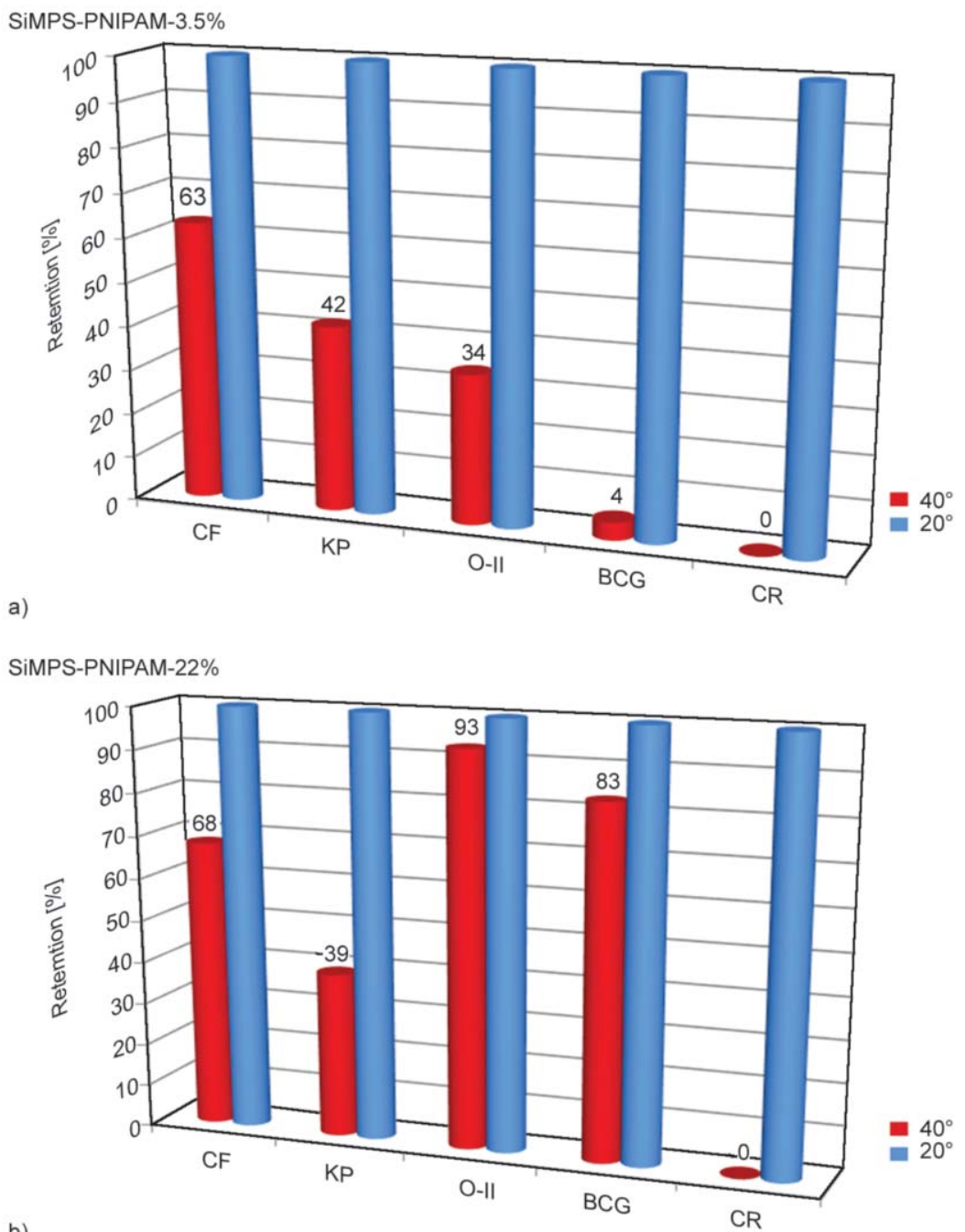

b)

Figure 8. Results of thermo-regulated elution of model compounds across hybrid microparticles beds at 20 and $40{ }^{\circ} \mathrm{C}$ by SiMPs-PNIPAM-3.5 (a) and 22\% (b) samples respectively. Note: 100\% retention refers to no elution and retention percentage at $40^{\circ} \mathrm{C}$ refers to concentrations of permeated solutions converted into percentages with respect to starting concentrations.

Table 1. Concentrations and retention percentages of model compounds across the thermoresponsive beds at $40^{\circ} \mathrm{C}$.

\begin{tabular}{|c|c|c|c|c|c|}
\hline \multicolumn{6}{|c|}{ SiMPs-PNIPAM-3.5\% } \\
\hline Compound & $\begin{array}{c}M_{\mathrm{w}} \\
{[\mathrm{g} / \mathrm{mol}]}\end{array}$ & $\begin{array}{c}\text { Starting conc. } \\
{[\mathrm{g} / \mathrm{l}]}\end{array}$ & $\begin{array}{c}\text { Eluted conc. } \\
{[\mathrm{g} / \mathrm{I}]}\end{array}$ & $\%$ in wlute, $x$ & $\begin{array}{l}\text { Retention } \\
\%(100-x)\end{array}$ \\
\hline Caffeine & 194.20 & $1.94 \cdot 10^{-3}$ & $7.24 \cdot 10^{-4}$ & 37 & 63 \\
\hline Ketoprofen & 254.28 & $1.42 \cdot 10^{-2}$ & $8.64 \cdot 10^{-3}$ & 38 & 42 \\
\hline Orange II & 350.32 & $3.50 \cdot 10^{-3}$ & $2.30 \cdot 10^{-3}$ & 66 & 34 \\
\hline Bromocresol green & 698.05 & $6.98 \cdot 10^{-4}$ & $6.60 \cdot 10^{-4}$ & 96 & 4 \\
\hline Cresol red & 382.43 & $9.60 \cdot 10^{-3}$ & $9.60 \cdot 10^{-3}$ & 100 & 0 \\
\hline \multicolumn{6}{|c|}{ SiMPs-PNIPAM-22\% } \\
\hline Compound & $\begin{array}{c}M_{\mathrm{w}} \\
{[\mathrm{g} / \mathrm{mol}]}\end{array}$ & $\begin{array}{l}\text { Starting conc. } \\
{[\mathrm{g} / \mathrm{l}]}\end{array}$ & $\begin{array}{c}\text { Eluted conc. } \\
{[\mathrm{g} / \mathrm{I}]}\end{array}$ & $\%$ in wlute, $x$ & $\begin{array}{l}\text { Retention } \\
\%(100-x)\end{array}$ \\
\hline Caffeine & 194.20 & $1.94 \cdot 10^{-3}$ & $6.14 \cdot 10^{-4}$ & 32 & 68 \\
\hline Ketoprofen & 254.28 & $1.42 \cdot 10^{-2}$ & $8.21 \cdot 10^{-3}$ & 61 & 39 \\
\hline Orange II & 350.32 & $3.50 \cdot 10^{-3}$ & $2.50 \cdot 10^{-4}$ & 7 & 93 \\
\hline Bromocresol green & 698.05 & $6.98 \cdot 10^{-4}$ & $1.23 \cdot 10^{-4}$ & 17 & 83 \\
\hline Cresol red & 382.43 & $9.60 \cdot 10^{-3}$ & $9.60 \cdot 10^{-3}$ & 100 & 0 \\
\hline
\end{tabular}


grafted on the surface of the vinylized silica microparticles (i.e. on the pores walls) is able to block water access to the pores or open it depending on the temperature. In facts, the mechanism depends not so much on the ability of PNIPAM to fill the pores when it is hydrated and to empty them when it collapses, but rather on the ability to screen or not the vinyl groups that impart superhydrophobicity to the surface of the particles. When PNIPAM chains are hydrated (at T $<$ LCST) they extend towards the solution, partially obstruct the pores and leave the vinyl groups exposed on the surface of the microparticles. On the contrary at T > LCST PNIPAN chains collapse, thus shielding the vinyl groups and making the pores of the packed bed water permeable.

The PNIPAM-grafting procedure, preparation of beds and permeability gating mechanism presented here can be potentially reproduced by using various oxide microparticles which are specifically chosen for the applications under study. Additionally, the hybrid thermoresponsive porous systems with thermally triggered gating mechanism developed here can be potentially used as component of advanced filtration or separation devices for thermo-regulated selective transport of solutions across a barrier or as flow regulators of aqueous solutions of compounds or simply water.

\section{Acknowledgements}

This work was supported by the Marie Sklodowska-Curie Research and Innovation Staff Exchange project funded by the European Commission H2020-MSCA-RISE-2014 [research project Mat4treaT, project number: 645551]. Authors thank Prof. A. Bianco Prevot and Dr. D. Fabbri for assistance in the preparation of the thermoresponsive cartridges.

\section{References}

[1] Browne T. E., Cohen Y.: Polymer-grafted silica: A screening system for polymeric adsorption resin development. Industrial and Engineering Chemistry Research, 32, 716-725 (1993).

https://doi.org/10.1021/ie00016a019

[2] Tripathi B. P., Dubey N. C., Simon F., Stamm M.: Thermo responsive ultrafiltration membranes of grafted poly( $N$-isopropyl acrylamide) via polydopamine. RSC Advances, 4, 34073-34083 (2014).

https://doi.org/10.1039/C4RA03485F
[3] Bilchak C. R., Buenning, E., Asai M., Zhang K., Durning C. J., Kumar S. K., Huang Y., Benicewicz B. C., Gidley D. W., Cheng S., Sokolov A. P., Minelli M., Doghieri F.: Polymer-grafted nanoparticle membranes with controllable free volume. Macromolecules, 50, 7111-7120 (2017). https://doi.org/10.1021/acs.macromol.7b01428

[4] Green E., Fullwood E., Selden J., Zharov I.: Functional membranes via nanoparticle self-assembly. Chemical Communications, 51, 7770-7780 (2015). https://doi.org/10.1039/C5CC01388G

[5] Liu L., Chen H., Yang F.: Enhancing membrane performance by blending ATRP grafted PMMA-TiO 2 or PMMA-PSBMA-TiO 2 in PVDF. Separation and $\mathrm{Pu}-$ rification Technology, 133, 22-31 (2014).

https://doi.org/10.1016/j.seppur.2014.06.015

[6] Fu Q., Rama Rao G. V., Ward T. L., Lu Y., Lopez G. P.: Thermoresponsive transport through ordered mesoporous silica/PNIPAAm copolymer membranes and microspheres. Langmuir, 23, 170-174 (2007).

https://doi.org/10.1021/la062770f

[7] Jadhav S. A., Brunella V., Scalarone D., Berlier G.: Poly(NIPAM-co-MPS)-grafted multimodal porous silica nanoparticles as reverse thermoresponsive drug delivery system. Asian Journal of Pharmaceutical Sciences, 12, 279-284 (2017).

https://doi.org/10.1016/j.ajps.2017.02.002

[8] Brunella V., Jadhav S. A., Miletto I., Berlier G., Ugazio E., Sapino S., Scalarone D.: Hybrid drug carriers with temperature-controlled on-off release: A simple and reliable synthesis of PNIPAM-functionalized mesoporous silica nanoparticles. Reactive and Functional Polymers, 98, 31-37 (2016).

https://doi.org/10.1016/j.reactfunctpolym.2015.11.006

[9] Ugazio E., Gastaldi L., Brunella V., Scalarone D., Jadhav S. A., Oliaro-Bosso S., Zonari D., Berlier G., Miletto I., Sapino S.: Thermoresponsive mesoporous silica nanoparticles as a carrier for skin delivery of quercetin. International Journal of Pharmaceutics, 511, 446-452 (2016).

https://doi.org/10.1016/j.ijpharm.2016.07.024

[10] Dutta K., De S.: Smart responsive materials for water purification: An overview. Journal of Material Chemistry A, 5, 22095-22112 (2017). https://doi.org/10.1039/C7TA07054C

[11] Zhao Q., Chen N., Zhao D., Lu X.: Thermoresponsive magnetic nanoparticles for seawater desalination. ACS Applied Materials and Interfaces, 5, 11453-11461 (2013). https://doi.org/10.1021/am403719s

[12] Ding J-J., Zhu J., Li Y-X, Liu X-Q., Sun L-B.: Smart adsorbents functionalized with thermoresponsive polymers for selective adsorption and energy-saving regeneration. Industrial and Engineering Chemistry Research, 56, 4341-4349 (2017).

https://doi.org/10.1021/acs.iecr.7b00582 
[13] Li P-F., Xie R., Jiang J-C., Meng T., Yang M., Ju X-J., Yang L., Chu L-Y.: Thermo-responsive gating membranes with controllable length and density of poly $(N-$ isopropylacrylamide) chains grafted by ATRP method. Journal of Membrane Science, 337, 310-317 (2009). https://doi.org/10.1016/j.memsci.2009.04.010

[14] Fu Q., Rao G .V. R., Ista L. K., Wu Y., Andrzejewski B. P., Sklar L. A., Ward T. L., López G. P.: Control of molecular transport through stimuli-responsive ordered mesoporous materials. Advanced Materials, 15, 1262 1266 (2003). https://doi.org/10.1002/adma.200305165

[15] Schepelina O., Zharov I.: PNIPAAM-modified nanoporous colloidal films with positive and negative temperature gating. Langmuir, 23, 12704-12709 (2007). https://doi.org/10.1021/la702008j

[16] Heskins M., Guillet J. E.: Solution properties of $\operatorname{poly}(N-$ isopropylacrylamide). Journal of Macromolecular Science Part A: Chemistry, 2, 1441-1455 (1968). https://doi.org/10.1080/10601326808051910

[17] Schild H. G.: Poly( $N$-isopropylacrylamide): Experiment, theory and application. Progress in Polymer Science, 17, 163-249 (1992). https://doi.org/10.1016/0079-6700(92)90023-R

[18] Halperin A., Kröger M., Winnik F. M.: Poly( $N$-isopropylacrylamide) phase diagrams: Fifty years of research. Angewantade Chemie International Edition, 54, 15342-15367 (2015).

https://doi.org/10.1002/anie.201506663

[19] Jadhav S. A., Nisticò R., Magnacca G., Scalarone D.: Packed hybrid silica nanoparticles as sorbents with thermo-switchable surface chemistry and pore size for fast extraction of environmental pollutants. RSC Advances, 8, 1246-1254 (2018).

https://doi.org/10.1039/c7ra11869d

[20] Abelow A. E., Zharov I.: Reversible nanovalves in inorganic materials. Journal of Materials Chemistry, 22, 21810-21818 (2012). https://doi.org/10.1039/c2jm33437b

[21] Chu L-Y.: Smart membrane materials and systems. Springer, Chengdu (2011). https://doi.org/10.1007/978-3-642-18114-6

[22] Meng T., Xie R., Chen Y-C., Cheng C-J., Li P-F., Ju X-J., Chu L-Y.: A thermo-responsive affinity membrane with nano-structured pores and grafted poly $(N$-isopropylacrylamide) surface layer for hydrophobic adsorption. Journal of Membrane Science, 349, 258-267 (2010). https://doi.org/10.1016/j.memsci.2009.11.058

[23] Li Y., Chu L-Y., Zhu J-H., Wang H. D., Xia S-L., Chen W-N.: Thermoresponsive gating characteristics of poly (n-isopropylacrylamide)-grafted porous poly(vinylidene fluoride) membranes. Industrial and Engineering Chemistry Research, 43, 2643-2649 (2004).

https://doi.org/10.1021/ie034334j
[24] Chu L-Y., Nitsuma T., Yamaguchi T., Nakao S-I.: Thermoresponsive transport through porous membranes with grafted PNIPAM gates. AIChE Journal, 49, 896-909 (2003).

https://doi.org/10.1002/aic.690490409

[25] Wang X-D., Shen Z-X., Sang T., Cheng X-B., Li M-F., Chen L-Y., Wang Z-S.: Preparation of spherical silica particles by Stöber process with high concentration of tetra-ethyl-orthosilicate. Journal of Colloid and Interface Science, 341, 23-29 (2010). https://doi.org/10.1016/j.jcis.2009.09.018

[26] Stöber W., Fink E. B., Bohn E.: Controlled growth of monodisperse silica spheres in the micron size range. Journal of Colloid and Interface Science, 26, 62-69 (1968). https://doi.org/10.1016/0021-9797(68)90272-5

[27] Liberman A., Mendez N., Trogler W. C., Kummel A. C.: Synthesis and surface functionalization of silica nanoparticles for nanomedicine. Surface Science Reports, 69, 132-158 (2014).

https://doi.org/10.1016/j.surfrep.2014.07.001

[28] van Blaaderen A., Vrij A.: Synthesis and characterization of monodisperse colloidal organo-silica spheres. Journal of Colloid and Interface Science, 156, 1-18 (1993).

https://doi.org/10.1006/jcis.1993.1073

[29] Choi H., Chen I-W.: Surface-modified silica colloid for diagnostic imaging. Journal of Colloid and Interface Science, 258, 435-437 (2003).

https://doi.org/10.1016/S0021-9797(02)00130-3

[30] Nozawa K., Gailhanou H., Raison L., Panizza P., Ushiki H., Sellier E., Delville J. P., Delville M. H.: Smart control of monodisperse stöber silica particles: Effect of reactant addition rate on growth process. Langmuir, 21, 1516-1523 (2005).

https://doi.org/10.1021/la048569r

[31] Lee Y-G., Park J-H., Oh C., Oh S-G., Kim Y. C.: Preparation of highly monodispersed hybrid silica spheres using a one-step sol-gel reaction in aqueous solution. Langmuir, 23, 10875-10878 (2007).

https://doi.org/10.1021/la702462b

[32] Chen S., Jiang X., Sun L.: Reaction mechanisms of $N$ isopropylacrylamide soap-free emulsion polymerization based on two different initiators. Journal of Macromolecular Science Part A: Pure and Applied Chemistry, 51, 447-455 (2014). https://doi.org/10.1080/10601325.2014.893144

[33] Virtanen O. L. J., Mourran A., Pinard P. T., Richtering W.: Persulfate initiated ultra-low cross-linked poly $(\mathrm{N}$ isopropylacrylamide) microgels possess an unusual inverted cross-linking structure. Soft Matter, 12, 39193928 (2016).

https://doi.org/10.1039/C6SM00140H

[34] Sanson N., Rieger J.: Synthesis of nanogels/microgels by conventional and controlled radical crosslinking copolymerization. Polymer Chemistry, 1, 965-977 (2010). https://doi.org/10.1039/c0py00010h 
[35] Xia L-W., Xie R., Ju X-J., Wang W., Chen Q., Chu L-Y.: Nano-structured smart hydrogels with rapid response and high elasticity. Nature Communications, 4, 2226/12226/11 (2013).

https://doi.org/10.1038/ncomms3226

[36] Lee Y-G., Park C-Y., Song K-H., Kim S-S., Oh S-G.: Preparation of monodispersed PNIPAm/silica composites and characterization of their thermal behaviors. Journal of Industrial and Engineering Chemistry, 18, 744-751 (2012).

https://doi.org/10.1016/j.jiec.2011.11.117

[37] Effati E., Pourabbas B.: One-pot synthesis of sub$50 \mathrm{~nm}$ vinyl- and acrylate-modified silica nanoparticles. Powder Technology, 219, 276-283 (2012). https://doi.org/10.1016/j.powtec.2011.12.062

[38] Li G., Shi Q., Yuan S. J., Neoh K. G., Kang E. T., Yang $\mathrm{X}$.: Alternating silica/polymer multilayer hybrid microspheres templates for double-shelled polymer and inorganic hollow microstructures. Chemistry of Materials, 22, 1309-1317 (2010).

https://doi.org/10.1021/cm903501e
[39] Jadhav S. A., Brunella V., Miletto I. Berlier G., Scalarone D.: Synthesis of poly( $N$-isopropylacrylamide $)$ by distillation precipitation polymerization and quantitative grafting on mesoporous silica. Journal of Applied Polymer Science, 133, 44181/1-44181/8 (2016). https://doi.org/10.1002/app.44181

[40] Jadhav S. A., Miletto I., Brunella V., Berlier G., Scalarone D.: Controlled post-synthesis grafting of thermoresponsive poly( $N$-isopropylacrylamide) on mesoporous silica nanoparticles. Polymers for Advanced Technology, 26, 1070-1075 (2015). https://doi.org/10.1002/pat.3534

[41] Jadhav S. A., Scalarone D., Brunella V., Ugazio E., Sapino S., Berlier G.: Thermoresponsive copolymergrafted SBA-15 porous silica particles for temperaturetriggered topical delivery systems. Express Polymer Letters, 11, 96-105 (2017). https://doi.org/10.3144/expresspolymlett.2017.11 